\title{
Incidência das dermatopatias auto-imunes em cães e gatos e estudo retrospectivo de 40 casos de lupus eritematoso discóide atendidos no serviço de dermatologia da Faculdade de Medicina Veterinária e Zootecnia da UNESP - Botucatu
}

\author{
Incidence of the autoimmune dermatosis in dogs and cats and \\ retrospective study of $\mathbf{4 0}$ cases of discoid lupus erythematosus \\ attended at the Dermatology Service of the College of Veterinary \\ Medicine and Animal Science of UNESP - Botucatu
}

\author{
Mariana Isa Poci Palumbo ${ }^{1 *}$; Luiz Henrique de Araújo Machado²; \\ Jorge Piovesan Conti $^{3}$; Fernanda Cristina de Oliveira ${ }^{4}$; Jéssica Correa Rodrigues ${ }^{5}$
}

Resumo

\begin{abstract}
O presente trabalho teve como objetivo fazer um levantamento da casuística de doenças dermatológicas auto-imunes e atualizar os dados referentes à ocorrência de lupus eritematoso discóide na população canina e felina atendida pelo Serviço de Dermatologia Veterinária da FMVZ-UNESP, campus de Botucatu, incluindo a espécie, sexo, raça, idade, localização e caracterização das lesões. Com a análise dos resultados, concluiu-se que a ordem de ocorrência de acordo com o número de casos de doenças dermatológicas auto-imunes nos animais atendidos pelo setor de dermatologia no período de 1988 a 2007 é: lupus eritematoso discóide, pênfigo foliáceo, síndrome úveo-dermatológica, pênfigo vulgar, lupus eritematoso sistêmico, eritema necrolítico migratório, eritema multiforme e pododermatite plasmocitária. Dos animais com lupus eritematoso discóide, todos eram da espécie canina, sendo a maioria fêmea e sem definição racial. As raças mais acometidas por lupus eritematoso discóide foram: Pastor Alemão e Akita e a média das idades foi de 56 meses. Notou-se que a maioria das lesões era localizada em plano nasal, narinas e região periocular. As lesões mais comumente observadas foram: crosta, despigmentação e eritema.
\end{abstract}

Palavras-chave: Doenças de pele auto-imunes, lupus eritematoso discóide, cães, gatos

\begin{abstract}
The objectives of this study were to do a survey of the autoimmune skin diseases and update the records regarding the occurrence of discoid lupus erythematosus in canine and feline populations attended
\end{abstract}

\footnotetext{
${ }^{1}$ Mestranda do Depto. de Clínica Veterinária - Universidade Estadual Paulista “Júlio de Mesquita Filho”- FMVZ-UNESP, Botucatu, Camilo Mazoni, 1055, apto. 42, bloco G, CEP: 18610-285, Botucatu, SP. E-mail: palumboma11@yahoo.com.br.

${ }^{2}$ Prof. Ass. do Depto. de Clínica Veterinária - Universidade Estadual Paulista "Júlio de Mesquita Filho"- FMVZ - UNESP Botucatu, Distrito de Rubião Jr. S/N. Botucatu - SP - Brasil, CEP 18618-000. E-mail: henrique@fmvz.unesp.br.

${ }^{3}$ Médico Veterinário Autônomo, Rua Pedro Angella n456, Vl. Pinheiro, CEP 18608-690, Botucatu-SP. E-mail: jpconti@gmail. com.

${ }^{4}$ Acadêmica do curso de Medicina Veterinária da Universidade Estadual Paulista "Júlio de Mesquita Filho"- FMVZ - UNESPBotucatu. Distrito de Rubião Jr. S/N. Botucatu - SP - Brasil, CEP 18618-000. E-mail: fernandacoliveira@msn.com

${ }^{5}$ Acadêmica do curso de Medicina Veterinária da Universidade Estadual Paulista "Júlio de Mesquita Filho"- FMVZ - UNESPBotucatu. Distrito de Rubião Jr. S/N. Botucatu - SP - Brasil, CEP 18618-000. E-mail: espertavet@yahoo.com.br

* Autor para correspondência
} 
at the Dermatology Service of the College of Veterinary Medicine and Animal Science of UNESP Botucatu, including species, gender, breed, age, location and characteristic of the lesions. Results have shown that the order of occurrence, regarding the number of cases of autoimmune skin diseases in the animals attended by the Dermatology Service in the period from 1988 to 2007 was: discoid lupus erythematosus, pemphigus folliaceus, uveo-dermatologic syndrome, pemphigus vulgaris, systemic lupus erythematosus, necrolytic migratory erythema, multiforme erythema and plasmacytic pododermatitis. All the animals with discoid lupus erythematosus were dogs and most of them were mongrel females. More frequently breeds affected by discoid lupus erythematosus were german shepherd and akita and the mean age was 56 months. Most lesions were located in nasal planum, narines and periocular area and were characterized by crusting, depigmentation and erythema.

Key words: Autoimmune skin diseases, discoid lupus erythematosus, dogs, cats

As dermatoses auto-imunes são bemidentificadas, mas são doenças raras de pele em cães e gatos (GROSS; IHRKE; WALDER, 1992). Nas doenças auto-imunes, os anticorpos ou linfócitos ativados são dirigidos contra as células do próprio organismo (BALDA et al., 2002). Apesar da fisiopatologia do desenvolvimento lesional nessas enfermidades estar bem determinada, não se sabe, ainda, o que estimularia tais anormalidades no sistema imunológico (WERNER, 1999). A predisposição genética parece ter um papel importante no desenvolvimento da auto-imunidade, mas múltiplos fatores devem estar envolvidos nessa alteração funcional (BALDA et al., 2002).

Em cães, o lupus eritematoso discóide (LED) é a segunda dermatopatia auto-imune mais comum, e usualmente é localizada no plano nasal (GERHAUSER; STROTHMANN-LVERSSEN; BAUMGORTNER, 2006). Quando comparada com o lupus eritematoso sistêmico, o LED é uma doença relativamente benigna, localizada na pele e membrana mucosa (SCOTT et al., 1983). O sinal clínico mais comum é a dermatite nasal, e os sinais podem piorar no verão (IWASAKI et al., 1995). LED não se torna doença generalizada, contudo as lesões podem se espalhar para a ponte nasal e, menos comumente, para região periocular, pina, membros distais e junções muco-cutâneas (lábios, cavidade oral e região genital) (GERHAUSER; STROTHMANN-LVERSSEN; BAUMGORTNER, 2006).
O diagnostico de LED é baseado nas alterações clínicas, histopatologia de fragmento de pele, imunofluorescência direta (IFD) ou teste de banda lúpica (SCOTT; MILLER; GRIFFIN, 2001). Tipicamente, a histopatologia mostra um infiltrado linficítico, macrofágico e plasmocítico com variáveis graus de edema intracelular (degeneração hidrópica) da camada de células basais, espessamento da membrana basal e celulas epidermais degeneradas (GROSS; IHRKE; WALDER, 1992). A terapia utiliza basicamente o uso de agentes imunossupressivos (WHITE, 1994). Normalmente utiliza-se a prednisona ou prednisolona $(1 \mathrm{mg} / \mathrm{Kg}$, $\mathrm{BID}, \mathrm{PO}$, com redução gradual até a mínima dose eficaz) (SCOTT; MILLER; GRIFFIN, 2001). Em raros casos, a gravidade da doença pode requerer uma terapia imunossupressiva mais potente, com azatioprina ou clorambucil (WHITE, 1994).

Os objetivos do presente trabalho foram fazer um levantamento da casuística de doenças dermatológicas auto-imunes e atualizar os dados referentes à ocorrência de lupus eritematoso discóide na população canina e felina, atendida pelo Serviço de Dermatologia Veterinária da FMVZUNESP campus de Botucatu, incluindo a espécie, sexo, raça, idade, localização e caracterização das lesões nos animais atendidos.

Todos os animais inclusos neste trabalho foram atendidos no Serviço de dermatologia do Hospital Veterinário da UNESP de Botucatu, no período de 1988 a 2007. Foram analisados todos os laudos de histopatologia compatíveis com dermatopatias 
auto-imunes, para determinar a incidência destas doenças em cães e gatos. Realizou-se também um estudo retrospectivo dos prontuários dos pacientes cuja biopsia de pele foi conclusiva para lupus eritematoso discóide para um levantamento de dados incluindo espécie, sexo, raça, idade, localização e caracterização das lesões no corpo do animal. Utilizou-se o teste de qui-quadrado para verificar a existência de diferença significativa entre os resultados obtidos. $\mathrm{O}$ nível de significância adotado foi de $5 \%$.

No período de 1988 a 2007, foram confirmados 66 casos de dermatopatias auto-imunes pelos resultados de exame histopatológico de lesões biopsiadas de cães e gatos. Os números de animais acometidos por cada doença foram: $40(60,6 \%)$ lupus eritematoso discóide, 11 (16,67\%) pênfigo foliáceo, 4 (6,06\%) síndrome úveo-dermatológica, 4 (6,06\%) pênfigo vulgar, $2(3,03 \%)$ lupus eritematoso sistêmico, 2 (3,03\%) eritema necrolítico migratório, 2 (3,03\%) eritema multiforme e $1(1,52 \%)$ pododermatite plasmocitária.

A análise das fichas dos animais com lupus eritematoso discóide mostrou que $100 \%$ dos animais eram da espécie canina, sendo 57,5\% fêmeas. Quanto à raça dos cães, observou-se que a maioria dos animais (60\%) não possuía raça definida. Dos 16 pacientes de raça definida, aqueles mais acometidos foram: Pastor Alemão (25\%) e Akita (25\%). Os oitos restantes pertenciam a raças distintas, incluindo: Bull terrier, Border Collie, Husky Siberiano, Teckel, Fox paulistinha, Dogue Alemão, Rottweiller e Collie. A média das idades foi de 56 meses, tendo variado de 3 a 132 meses. A lesões localizavam-se mais freqüentemente em: plano nasal (85\%), narina (47,5\%), região periocular (47,5\%), comissura labial $(30 \%)$, membros $(22,5 \%)$, coxin $(12,5 \%)$, escroto (12,5\%) e prepúcio (12,5\%). Outras localizações que apareceram em menor porcentagem são: cauda, interdígito, pavilhão auricular, palato mole, vulva, axilas e região perianal. As lesões observadas nos cães foram: crosta (72,5\%), despigmentação $(67,5 \%)$, eritema $(60 \%)$, alopecia $(35 \%)$, ulceração
(30\%), exsudação (15\%), hiperqueratose $(12,5 \%)$ e pápula $(7,5 \%)$.

Sabe-se que as dermatopatias auto-imunes correspondem a 1,0-1,5\% da casuística de atendimento dos serviços de referência em dermatologia (SCOTT; MILLER; GRIFFIN, 2001). Em 20 anos foram confirmados 66 casos de dermatopatias auto-imunes em cães e gatos atendidos pelo setor de dermatologia da UNESPBotucatu. Segundo Bennion e Norris (1997), LED é uma das doenças de pele auto-imune mais comumente observada em cães e gatos, e acometeu $60,6 \%$ dos animais inclusos no presente estudo. Muitos autores acreditam que o pênfigo foliáceo é a dermatopatia auto-imune mais freqüentemente observada em cães e gatos, ficando aquém ou muito próximo, em termos de ocorrência, à casuística de lúpus eritematoso (SCOTT, 1987). Segundo Balda et al. (2008), o pênfigo foliáceo é considerado a forma mais comum das doenças do complexo pênfigo, fato também observado neste levantamento de dados.

Casos de LED canino têm sido relatados desde 1979, quando Griffin descreveu o primeiro caso. O LED tem sido relatado em gatos, mas é aparentemente incomum (WILLEMSE; KOEMAN, 1989). Neste levantamento de dados, $100 \%$ dos animais com LED eram da espécie canina. Provavelmente não existe predileção por sexo, porque tanto fêmeas (SCOTT et al., 1983) como machos (OLIVRY; ALHAIDARI; CARLOTTI, 1987) foram relatados como predispostos. Neste trabalho, 57,5\% dos animais eram fêmeas.

A maioria dos cães este estudo não possuíam definição racial. Segundo Olivry, Alhaidari e Carlotti (1987), Scott (1987) e Scott et al. (1983), os Collies, Pastores Alemães, Shetland Sheepdogs, Huskies siberianos, Brittany Spaniels e Pointers Alemães de pêlo curto demonstraram predisposição. Dos 16 animais de raça, notou-se que Pastor Alemão e Akita foram mais acometidos. Goo et al. (2008) relataram a ocorrência de lupus eritematoso discóide em uma cadela Spitz de 3 anos de idade, com lesões cutâneas 
atípicas, incluindo eritema difuso e descamação em região de tronco dorsal, sem a presença de lesões em face.

Segundo Goo et al. (2008), num primeiro momento, as lesões de LED são usualmente localizadas na junção dorsal do plano nasal e pele. As lesões também podem se desenvolver na região periocular e na pina da orelha como um padrão bilateral simétrico (GRIFFIN et al., 1979; JACKSON et al., 2004), na pele perioral, e na cavidade oral (YAGER; WILCOCK, 1994). Raramente, as lesões podem ser encontradas no tronco, membros, pés, genitais e região perianal (DAY; HANLON; POWELL, 1993; GERHAUSER; STROTHMANN-LVERSSEN; BAUMGORTNER, 2006; GRIFFIN et al., 1979; YAGER; WILCOCK, 1994). No presente estudo, as lesões localizaramse mais freqüentemente em plano nasal, narina, periocular, comissura labial, membros, coxim, escroto e prepúcio. Gerhauser, Strothmann-Lverssen e Baumgortner (2006) relataram a ocorrência de LED em um cão da raça Bavarian Mountain Scenthound com lesões cutâneas erosivas crônicas localizadas exclusivamente na região perianal, sem o envolvimento de face.

No presente estudo, a média das idades foi de 56 meses e, segundo Scott, Miller e Griffin (2001), nenhuma predileção por idade foi relatada. Usualmente, o LED causa despigmentação, eritema, úlcera e crostas ocasionais (WHITE, 1994; GERHAUSER; STROTHMANN-LVERSSEN; BAUMGORTNER, 2006). As lesões observadas nos cães neste levantamento de dados foram: crosta, despigmentação, eritema, alopecia, ulceração, exsudação, hiperqueratose e pápula. Segundo Gross et al. (2005), as lesões iniciais são caracterizadas por despigmentação, eritema, descamação de padrão bilateral simétrico.

Através da análise dos resultados, concluiu-se que a ordem de ocorrência de doenças dermatológicas auto-imunes nos animais atendidos pelo setor de dermatologia no período de 1988 a 2007 é: lupus eritematoso discóide, pênfigo foliáceo, síndrome úveo-dermatológica, pênfigo vulgar, lupus eritematososistêmico,eritemanecrolíticomigratório, eritema multiforme e pododermatite plasmocitária. Dos animais com lupus eritematoso discóide, todos eram da espécie canina, sendo a maioria fêmea e sem definição racial. Notou-se que a maioria das lesões era localizada em plano nasal, narinas e região periocular. As lesões mais comumente observadas foram: crosta, despigmentação e eritema.

\section{Referências}

BALDA, A. C.; IKEDA, M. O.; LARSSON JUNIOR, C. E.; MICHALANY, N. S.; LARSSON, C. E. Pênfigo foliáceo canino: estudo retrospectivo de 43 casos clínicos e terapia (2000-2005). Pesquisa Veterinária Brasileira, Rio de Janeiro, v. 28, n. 8, p. 387-392, 2008.

BALDA, A. C.; OTSUKA, M.; MICHALANY, N. S.; LARSSON, C. E. Pênfigo foliáceo em cães: levantamento retrospectivo de casos atendidos no período de novembro de 1986 a julho de 2000 e de resposta aos protocolos de terapia empregados no Hospital Veterinário da USP. Revista Brasileira de Ciência Veterinária, Rio de Janeiro, v. 9, n. 2, p. 97-101, 2002.

BENNION, S. D.; NORRIS, D. A. Ultraviolet light modulation of autoantigens, epidermal cytokines and adhesion molecules as contributing factors of the pathogenesis of cutaneous LE. Lupu, London, v. 6, n. 2, p. 181-192, 1997.

DAY, M. J.; HANLON, L.; POWELL, L. M. Immunemediated skin disease in the dog and cat. Journal of Comparative Pathology, Edinburgh, v. 109, n. 4, p. $395-$ 407, 1993.

GERHAUSER, I.; STROTHMANN-LVERSSEN, A.; BAUMGORTNER, W. A case of interface perianal dermatitis in a dog: is this an unusual manifestation of lupus erythematosus? Veterinary Pathology, Washington, v. 43, n. 5, p. 761-764, 2006.

GOO, M. J.; PARK, J. K.; HONG, I. H.; YANG, H. J.; YUA, D. W.; KI, M. R.; HAN, J. Y.; JI, A. R.; KIM, T. H.; WILLIAMS, B. H.; JEONG, K. S. Discoid Lupus Erythematosus (DLE) in a Spitz Dog. Journal of Veterinary Medical Science, Tokyo, v. 70, n. 6, p. 633635, 2008 . 
GRIFFIN, C. E.; STANNARD, A. A.; IHRKE, P. J.; ARDANS, A. A.; CELLO, R. M.; BJORLING, D. R. Canine discoid lupus erythematosus. Veterinary Immunology Immunopathology, Amsterdam, v. 1, n. 1, p. 79-87, 1979.

GROSS, T. L.; IHRKE, P. J.; WALDER, E. Veterinary dermatopathology: a macroscopic and microscopic evaluation of canine and feline skin disease. St Louis: Mosby Year Book, 1992.

GROSS, T.L.; IHRKE, P. J.; WALDER, E. J.;AFFOLTER, V. K. Interface diseases of the dermal-epidermal junction. In: . Skin diseases of the dog and cat: clinical and histopathologic diagnosis. 2. ed. Oxford: Blackwell Science Ltd, 2005. p. 49-70.

IWASAKI, T.; SHIMIZU, M.; OBATA, H.; YANAI, T.; KITAGAWA, H.; SASAKI, Y. A canine case of discoid lupus erythematosus with circulating autoantibody. Journal of Veterinary Medical Science, Tokyo, v. 57, n. 6, p. 1097-1099, 1995.

JACKSON, H. A.; OLIVRY, T.; BERQET, F.; DUNSTON, S. M.; BONNEFONT, C.; CHABANNE, L. Immunopathology of vesicular cutaneous lupus erythematosus in the rough collie and Shetland sheepdog: a canine homologue of subacute cutaneous lupus erythematosus in humans. Veterinary Dermatology, Oxford, v. 4, n. 15, p. 230-239, 2004.

OLIVRY, T.; ALHAIDARI, Z.; CARLOTTI, D.; GUAGUÈRE, E.; RÉGNIER, A.; HUBERT, B.; MAGNOL, J.P.; OKSAM, F. Le lupus érythémateux discoide du chien: à propos de 22 observations. Pratique Médicale et Chirurgicale de l'Animal de Compagnie, $\mathrm{n}$. 22, p. 205-214, 1987.

SCOTT, D. W. Immune-mediated dermatoses in domestic animals: Ten years after. Part I. Compendium on Continuing Education for the practicing Veterinarian, Princeton, v. 9, n. 1, p. 424-435, 1987.

SCOTT, D. W.; MILLER, W. H.; GRIFFIN, C. E. Immune-mediated skin disorders. In:_. Small

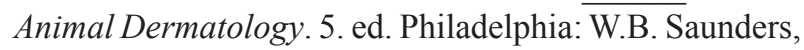
2001. p. 667-779.

SCOTT, D. W.; NALTON, D. K.; MANNING, T. O.; SMITH, C. A.; LEWIS, R. M. Canine lupus erythematosus. II: Discoid lupus erythematosus. Journal of American Animal Hospital Association, Lakewood, v. 1, n. 19, p. 482-488, 1983.

WERNER, A. H. Recognizing and treating discoid lupus erythematous and pemphigus foliaceus in dog. Veterinary Medicine, Chicago, v. 1, n. 94, p. 955-966, 1999.

WHITE, S. D. Diseases of the nasal planum. Veterinary Clinics of North America: Small Animal Practice, Philadelphia, v. 24, n. 5, p. 887-895, 1994.

WILlEMSE, T.; KOEMAN, J. P. Discoid lupus erythematosus in cats. Veterinary Dermatology, Oxford, v.1, n. 1, p. 19-24, 1989.

YAGER, J. A.; WILCOCK, B. P. Interface dermatitis. In: . Color atlas and text of surgical pathology of the dog and cat. London: Mosby-Years Book, 1994. v. 1, p. 92-95. 
